Ciência Florestal, Santa Maria, v. 23, n. 1, p. 117-127, jan.-mar., 2013

ISSN 0103-9954

\title{
MODELAGEM DO CARBONO ORGÂNICO TOTAL E DA LENHA POR UNIDADE DE ÁREA PARA BRACATINGAIS NATIVOS
}

\author{
MODELING FOR TOTAL AND FIREWOOD ORGANIC CARBON PER UNIT OF AREA FOR \\ NATIVE Mimosa scabrella STANDS
}

\author{
Ronan Felipe de Souza ${ }^{1}$ Sebastião do Amaral Machado ${ }^{2}$ Afonso Figueiredo Filho ${ }^{3}$ \\ Henrique Soares Koehler ${ }^{4}$
}

\begin{abstract}
RESUMO
O conceito de fixação de carbono normalmente se relaciona com a ideia de armazenar reservas de carbono em solos, florestas e outros tipos de vegetação. A presente pesquisa objetivou a modelagem do carbono orgânico total da parte aérea e da lenha por unidade de área fixada em povoamentos de bracatinga (Mimosa scabrella Bentham) na região metropolitana de Curitiba. Os dados são provenientes de 272 parcelas temporárias com idades variando de 3 a 18 anos. Foram testados 21 modelos tradicionais: 10 aritméticos e 11 logarítmicos; foram também desenvolvidos modelos pelo processo Stepwise a partir de uma matriz de correlação. As equações ajustadas foram comparadas pelo coeficiente de determinação ajustado $\left(\mathrm{R}_{\mathrm{aj}}^{2}\right)$, erro padrão da estimativa percentual $\left(\mathrm{S}_{\mathrm{yx}} \%\right)$, teste $\mathrm{F}$ e distribuição gráfica de resíduos. A correlação linear simples entre as variáveis utilizadas diferenciou-se daqueles resultados tradicionalmente encontrados para o volume dos povoamentos. Este resultado foi também constatado no ajuste dos modelos matemáticos por Stepwise, nos quais, a variável altura total média não compôs nenhum dos modelos ajustados. Apesar de eficientes, as equações tradicionais selecionadas mostraram-se menos acuradas que as equações ajustadas por Stepwise, reforçando a importância e a melhoria que este método representa para estudos de modelagem.
\end{abstract}

Palavras-chave: bracatinga; carbono orgânico estocado; equações estimativas.

\begin{abstract}
The fixed carbon concept is normally related to the idea of storing carbon in forest soil and other vegetations type. This research aimed the modeling of total above ground organic carbon and also for the firewood component by unit of area fixed in Mimosa scabrella stands in the metropolitan region of Curitiba. The database came from 272 temporary sample plots with ages ranging from 3 to 18 years. It was tested 21 traditional models: 10 arithmetic ones and 11 logarithmic ones; it was also developed models by Stepwise process from a correlation matrix. The equation of best performance was chosen based on $\mathrm{R}^{2} \mathrm{aj}, \mathrm{Syx} \%, \mathrm{~F}$ value and on graphic analysis of residuals. A simple linear correlation between the used variables differed from those traditionally found results for volume of stands. This result was also observed in the fitting of mathematical models by stepwise, in which the variable average height didn't compose any adjusted model. Although efficient, selected traditional equations were less accurate than the equations adjusted by stepwise, reinforcing the importance and improving that this method represents to modeling studies.
\end{abstract}

Keywords: Mimosa scabrella; stocked organic carbon; estimative equations.

1. Engenheiro Florestal, Msc., Doutorando no Programa de Pós-graduação em Engenharia Florestal, Universidade Federal do Paraná, Rua Lothário Meissner, 632, CEP 80210-170, Curitiba (PR). ronanflorestal@gmail.com

2. Engenheiro Florestal, PhD., Professor Sênior da Universidade Federal do Paraná, Rua Lothário Meissner, 632, CEP 80210-170, Curitiba (PR). Pesquisador1A do CNPQ. samachado@ufpr.br

3. Engenheiro Florestal, Dr., Professor Adjunto da Universidade do Centro Oeste e Professor Sênior da Universidade Federal do Paraná, Rua Lothário Meissner, 632, CEP 80210-170, Curitiba (PR). Pesquisador 1C do CNPq. afig@floresta.ufpr.br

4. Engenheiro Florestal, Dr., Professor Adjunto da Universidade Federal do Paraná, Rua Lothário Meissner, 632, CEP 80210-170, Curitiba (PR). Pesquisador 2 do CNPq. koehler@agrarias.ufpr.br

Recebido para publicação em 17/09/2010 e aceito em 16/11/2011 


\section{INTRODUÇÃO}

A bracatinga (Mimosa scabrella Bentham) é uma espécie pioneira, heliófila, nativa da Floresta Ombrófila Mista com dominância de Araucaria angustifolia. Esta espécie tem sido amplamente cultivada por pequenos e médios agricultores da região metropolitana de Curitiba desde 1909, quando Romário Martins, após identificar a simplicidade e o baixo custo das práticas silviculturais necessárias à sua implantação e manutenção, sugeriu a utilização dessa espécie (CARPANEZZI et al., 1988). Atualmente, a área plantada de bracatinga tem diminuído, entretanto, o sistema tradicional de cultivo ainda é uma atividade de grande importância econômica para pequenos e médios proprietários da região e que dificilmente será erradicado.

A contínua e crescente emissão do carbono na sua forma gasosa $\left(\mathrm{CO}_{2}\right)$ vem sendo condenada e combatida por diversos governos e entidades sociais. Vários esforços vêm sendo feitos para minimizar essa questão, dentre eles está a aprovação do Protocolo de Quioto que estabeleceu que os países desenvolvidos reduzissem suas emissões de $\mathrm{CO}_{2}$. Outro ponto muito importante, abordado nesse documento, foi a comercialização de créditos de carbono. Propiciando que países, ou mesmo empresas que fiquem abaixo de suas metas de lançamento de $\mathrm{CO}_{2}$ possam vender créditos para outras empresas ou para outros países que ultrapassam suas metas (GORGENS et al., 2005). Desta forma, poderse-ia atingir o balanceamento das emissões de $\mathrm{CO}_{2}$ e compensar-se-iam os esforços daqueles que reduziram o lançamento desse gás na atmosfera.

Vários pesquisadores vêm trabalhando no sentido de ampliar os conhecimentos sobre a captura de $\mathrm{CO}_{2}$ por meio de reflorestamentos e florestamentos e que, em teoria, pode ser quantificado estimandose o armazenamento de carbono atmosférico na biomassa e no solo da floresta (BALBINOT et al., 2003).

Schneider et al. (2005) quantificaram o carbono estocado na biomassa de povoamentos de Acácia-Negra (Acacia mearnsii De Wild.). Em seus resultados esses autores destacaram que a concentração de carbono na biomassa total média foi de $40 \%$.

Almeida et al. (2010) efetuaram estimativa do carbono em povoamentos de Teca (Tectona grandis L.f.). A quantidade média de carbono encontrada por hectare foi de $104,3 \mathrm{t}$ de $\mathrm{C}$ aos 1,5 anos, 180,8 t de $\mathrm{C}$ aos 2,5 anos, 303,1 t de $\mathrm{C}$ aos
3,5 anos e de 322,3 t de $\mathrm{C}$ aos 5,5 anos, considerando o número de plantas por hectare nas diferentes idades. Esses autores identificaram nos resultados que a distribuição média de carbono por componente no sistema foi de $90,8 \%$ no solo, $5,04 \%$ para a parte aérea, 3,04\% nas raízes e de $1,21 \%$ na serapilheira acumulada.

Rochadelli (2001) estudou a estrutura de fixação de carbono em reflorestamentos de bracatinga encontrando teores relativos de carbono orgânico fixado na biomassa total entre 40 e $45 \%$. Watzlawick et al. (2004) avaliaram os teores de carbono em espécies da Floresta Ombrófila Mista. Estes autores encontraram os seguintes teores de carbono em porcentagem para a bracatinga de 44,12, $40,9,41,9,37,3$ e 41,3 para folhas, galhos vivos, galhos mortos, casca e fuste, respectivamente.

Weber et al. (2006) encontraram valores percentuais de carbono fixado na biomassa da bracatinga iguais a: $44,9,40,9,41,9,37,2,41,3$ para folhas, galhos vivos, galhos mortos, casca e fuste, respectivamente. Esses autores destacaram em seus resultados que os valores percentuais encontrados foram muito semelhantes aos de Watzlawick et al. (2004).

Urbano et al. (2008), trabalhando com bracatingais da região metropolitana de Curitiba, quantificaram o carbono orgânico fixado na biomassa, esses autores também ajustaram modelos para estimar o carbono orgânico em compartimentos das árvores de bracatinga. Seus resultados revelaram uma maior concentração no componente folhagem $(45,46 \%)$, seguida pela casca $(44,69 \%)$, galhos menores que $4 \mathrm{~cm}(44,14 \%)$, galhos maiores que $4 \mathrm{~cm}(43,95 \%)$ e por fim, o fuste $(43,69 \%)$. As equações ajustadas para estimar o carbono total, do fuste e para a lenha foram consideradas adequadas, enquanto as equações para galhos e folhagem não apresentaram resultados satisfatórios.

Em relação à modelagem por unidade de área, vários são os trabalhos encontrados na literatura, dentre os quais estão o de Figueiredo Filho et al. (1982); Rosot et al. (1990); Silva (1995); Oliveira et al. (2005) e Machado et al. (2008). No entanto, não são encontrados trabalhos na literatura referentes à modelagem por unidade de área para 0 carbono orgânico.

Dentre os estudos de modelagem por unidade de área citados está o de Machado et al. (2008), que desenvolveram equações para estimativas do volume por unidade de área para bracatingais da região metropolitana de Curitiba. Os resultados 
revelaram alta correlação das variáveis combinadas $\mathrm{G} \bar{h}$ (área basal x altura total média) e $\mathrm{Gh}_{\text {dom }}$ (área basal $\mathrm{x}$ altura dominante) com o volume total e da lenha nas formas aritmética e logarítmica, sendo estas as variáveis incluídas no modelo Stepwise, desenvolvido por esses autores. Dos modelos aritméticos e semilogarítmicos selecionados da literatura os que apresentaram melhores ajustes foram aqueles que envolveram a variável $G \bar{h}$, sendo considerados inadequados aqueles que não continham esta variável. Os modelos logarítmicos apresentaram melhores ajustes do que os aritméticos, sempre apresentando em sua estrutura as variáveis $\mathrm{G} \bar{h}$ e $\mathrm{Gh}_{\text {dom }}$ na forma logaritmizada.

Dando sequência à pesquisa desenvolvida por Urbano et al. (2008) a presente pesquisa objetivou a modelagem do carbono orgânico total da parte aérea e da lenha por unidade de área fixado em povoamentos de bracatinga (Mimosa scabrella Bentham) na região metropolitana de Curitiba.

\section{MATERIAL E MÉTODOS}

\section{A área de estudo}

A região metropolitana de Curitiba situase no primeiro planalto do estado do Paraná. As faces leste, norte e sul são fortemente influenciadas pela Serra do Mar, já na região oeste, por ser mais interiorizada, apresenta influência da Escarpa Devoniana. A região norte é conhecida como Vale do Ribeira, local onde foi coletado o maior volume de dados para este trabalho. A altitude da área de estudo varia de $850 \mathrm{~m}$ em Agudos do Sul até $980 \mathrm{~m}$ acima do nível do mar em Bocaiúva do Sul.

O clima, de acordo com a classificação de Köppen, é denominado $\mathrm{Cfb}$, com temperatura média anual de $16,5{ }^{\circ} \mathrm{C}$, temperatura do mês mais quente de $20,4{ }^{\circ} \mathrm{C}$ e do mês mais frio de $12,7^{\circ} \mathrm{C}$. A maior precipitação pluviométrica ocorre em janeiro com 190,7 mm e a menor em agosto com $78,2 \mathrm{~mm}$; A precipitação média anual fica em torno de 1.500 milímetros (MAACK, 2002). Em relação à vegetação, a região caracteriza-se pela transição entre a Floresta Ombrófila Densa e a Floresta Ombrófila Mista, com dominância de Araucaria angustifolia.

A geologia da região é de origem metamórfica o que proporciona uma topografia fortemente ondulada com solos muito heterogêneos no que se refere à profundidade e fertilidade. Os solos são predominantemente Cambissolos álicos e apresentam-se pobres, pouco desenvolvidos, ácidos, com teores elevados de alumínio e baixa saturação de bases (CARPANEZZI et al., 1988).

\section{Base de dados}

A base de dados para o desenvolvimento desta pesquisa compôs-se de 272 parcelas temporárias, das quais, 17 parcelas foram extraídas para a validação das equações ajustadas selecionadas como melhores. Desta forma a base de dados final, utilizada para os ajustes dos modelos para estimar o carbono orgânico total da parte aérea e da lenha por unidade de área foi de 255 parcelas, para a qual foi realizada uma análise estatística de cada variável, bem como o cálculo do número de amostras necessárias em função da variância da população; a precisão requerida foi de $10 \%$ com probabilidade fixada em $95 \%$.

Foram mensurados todos os indivíduos nas unidades amostrais, utilizando-se vara graduada para medir altura total e altura de fuste e fita métrica para medir diâmetro a altura do peito.

As estimativas do carbono orgânico total da parte aérea e da lenha de cada indivíduo dentro das parcelas foram feitas por meio das respectivas equações ajustadas por Urbano et al. (2008), as quais podem ser observadas a seguir com suas estatísticas de ajuste, (1) e (2). A soma do carbono orgânico de todos os indivíduos de cada parcela resultou no carbono orgânico em cada unidade de área. Esses valores foram extrapolados para o hectare tal como se usa nos inventários volumétricos convencionais partindo de equações de volume.

$$
\begin{gathered}
\mathrm{CT}_{\mathrm{i}}=-4,08046+0,20748 \mathrm{dap}^{2} \\
\mathrm{R}_{\text {aj }}^{2}=0,9490 \\
\mathrm{~S}_{\mathrm{yx}} \%=24,44 \\
\mathrm{CL}_{\mathrm{i}}=-4,85369+0,18859 \mathrm{dap}^{2} \\
\mathrm{R}_{\text {aj }}^{2}=0,9530 \\
\mathrm{~S}_{\mathrm{yx}} \%=24,04
\end{gathered}
$$

Em que:

$\mathrm{CT}_{\mathrm{i}}=$ Carbono orgânico total individual $(\mathrm{kg}) ; \mathrm{CL}_{\mathrm{i}}=$ Carbono orgânico da lenha individual $(\mathrm{kg})$; dap = Diâmetro a altura do peito $(\mathrm{cm})$.

Foram também determinados a área basal e o número de árvores por hectare para cada unidade amostral, bem como a idade, índice de sítio, altura total média, altura dominante, altura de fuste média, diâmetro médio e diâmetro quadrático de cada parcela. Estas variáveis foram utilizadas na matriz de correlação. 
A classificação de sítios das parcelas foi realizada com base na classificação de sítios para bracatingais da região metropolitana de Curitiba realizada por Machado et al. (2011). Esses autores definiram para tanto, 3 classes de sítio com amplitude de 4 metros na idade índice de 7 anos: I, II e III, com 17, 13 e 9 m, respectivamente.

\section{Modelos tradicionais testados}

Dos 21 modelos testados, 10 foram aritméticos e semilogarítmicos, identificados de a1 até a10 e; 11 foram logarítmicos, identificados de $\ln 1$ a $\ln 11$. Visando à correção da discrepância logarítmica, para aqueles modelos logaritmizados, foi efetuado o recálculo das estatísticas de ajuste aplicando-se o Fator de Correção de Meyer (FCM).

\section{A matriz de correlação linear simples e os modelos por Stepwise}

A análise da correlação entre as variáveis independentes e o peso de carbono orgânico total da parte aérea e da lenha, na forma pura e logaritmizada, foi realizada por meio de uma matriz de correlação linear simples. O conjunto de variáveis independentes totalizou 85 , entre variáveis puras, inversas, combinadas, potencializadas e logaritmizadas.

A partir da matriz de correlação linear simples foram construídos dois modelos para cada variável dependente pelo processo Stepwise: um com a variável dependente na forma pura e outro na forma logaritmizada. O método de Stepwise consiste na inclusão de variáveis independentes em ordem decrescente de correlação linear simples com a variável dependente e que, quando não resultam em melhora significativa do modelo são eliminados. O nível de significância utilizado para a construção dos modelos desta modalidade foi de $95 \%$.

\section{Seleção de equações}

Para definição da melhor equação estimativa para cada variável dependente foram efetuadas comparações das estatísticas de ajuste e precisão: coeficiente de determinação ajustado $\left(\mathrm{R}^{2}{ }_{\mathrm{aj}}\right)$, erro padrão da estimativa percentual $\left(\mathrm{S}_{\mathrm{yx}} \%\right)$, teste $\mathrm{F}$ e distribuição gráfica de resíduos percentual. Para aquelas equações onde a variável dependente foi logaritmizada ou transformada, efetuou-se o cálculo do Índice de Schlaegel (IA), compatível com o valor de coeficiente de determinação dos demais modelos e $\mathrm{S}_{\mathrm{yx}} \%$ recalculado pelo Fator de
Correção de Meyer (FCM).

\section{Validação das equações selecionadas}

Para que fossem consideradas válidas as equações selecionadas para cada caso, adotou-se o teste de aderência de Qui-quadrado $\left(x^{2}\right)$, ao nível de $95 \%$ de significância. Foi realizada a análise da distribuição de resíduos para as 17 parcelas retiradas da base de dados, servindo também como critério de validação.

\section{RESULTADOS E DISCUSSÃO}

\section{Caracterização da base de dados}

Os resultados para a suficiência amostral demonstraram que a base de dados utilizada foi eficiente para a estimativa de todas as variáveis; é possível afirmar que a mesma apresentou-se sólida, representativa das diferentes combinações de idades e sítios existentes na região metropolitana de Curitiba. A variável $\mathrm{N}$ (número de árvores por hectare) foi responsável pelo maior número de amostras necessárias, com 205 amostras, inferior à amostragem que foi realizada para o desenvolvimento do estudo.

Para as variáveis dependentes os coeficientes de variação $(\mathrm{CV} \%)$ foram inferiores a $40 \%$; a variável independente que apresentou o maior valor foi o número de indivíduos $(72,72 \%)$ e a variável que apresentou menor valor foi a altura total média $(19,71 \%)$. Este resultado foi justificado pela menor influência que a altura total média sofre pela densidade, resultando em curvas de produção estáveis.

O carbono orgânico total da parte aérea apresentou o maior peso em um povoamento de 6 anos, no sítio II, comvaloriguala $48.238,09 \mathrm{~kg} \cdot \mathrm{ha}^{-1}$; o menor peso foi de $5.136,78 \mathrm{~kg} \cdot \mathrm{ha}^{-1}$, observado para um povoamento com 14 anos no sítio III. A amplitude foi de $43.679,22 \mathrm{~kg} \mathrm{ha}^{-1}$. O maior peso do carbono orgânico da lenha foi de 41.410, $74 \mathrm{~kg} \cdot \mathrm{ha}^{-1}$, observado no sítio I em um povoamento de 7 anos; o menor peso foi igual a 4.583,24 kg.ha-1, observado em um povoamento de 14 anos no sítio III. A amplitude foi de $36.827,50 \mathrm{~kg} \cdot \mathrm{ha}^{-1}$.

Schneider et al. (2005) trabalhando com Acácia-Negra (Acacia mearnsii De Wild.) encontraram valores um pouco superiores aos observados para a bracatinga no presente trabalho. Em seus resultados esses autores destacaram que o estoque de carbono estimado pelo método de derivação de volume em carbono, em povoamentos 
de 7 anos de idade, foi de 99,46 t.ha ${ }^{-1}$ no índice de sítio 20,82,98 t.ha ${ }^{-1}$ no índice de sítio $16 \mathrm{e}$ 46,13 t.ha ${ }^{-1}$ no índice de sítio 12. Esses resultados, no entanto, incluíram também o carbono fixado na serrapilheira e nas raízes das plantas.

\section{Matriz de correlação linear simples}

Na Tabela 1 são apresentadas as variáveis independentes que mostraram correlação linear simples (r) superior a 0,9 para, pelo menos, uma das variáveis dependentes. Foram destacadas as duas maiores correlações para cada variável.

As variáveis independentes que em geral apresentam as maiores correlações com o volume por unidade de área são aquelas que envolvem a área basal combinada com a altura total média ou com a altura dominante (FIGUEIREDO FILHO et al., 1982; ROSOT et al., 1990; SILVA, 1995; OLIVEIRA et al., 2005; MACHADO et al., 2008). Esse resultado, entretanto, não foi observado nesta pesquisa para o carbono orgânico total da parte aérea e da lenha por unidade de área, para os quais as variáveis mais correlacionadas envolveram a área basal combinada com o diâmetro médio ou diâmetro quadrático.

Urbano et al. (2008), quando desenvolveram modelos individuais para a estimativa do carbono orgânico total da parte aérea e da lenha da bracatinga, observaram que, a correlação com a altura não era significativa, eliminando então essa variável das equações selecionadas para utilização.

Machado et al. (2008), trabalhando com o volume do povoamento de bracatinga, mencionaram que as variáveis puras, exceto a área basal, apresentaram baixa correlação com a variável dependente, e que, quando combinadas apresentaram elevadas correlações. No presente estudo esse resultado também foi observado, no entanto, envolvendo também as variáveis diâmetro médio $(\overline{\mathrm{d}})$ e diâmetro quadrático $\left(\mathrm{d}_{\mathrm{g}}\right)$, como pode ser observado na Tabela 1.

\section{Equações estimativas do carbono orgânico total da parte aérea}

Os coeficientes com respectivas estatísticas de ajuste e precisão das equações tradicionais ajustadas para estimativa do carbono orgânico total da parte aérea são apresentados na Tabela 2. Em geral, os resultados obtidos para as equações foram satisfatórios, com altos valores de $\mathrm{R}^{2}{ }_{\text {aj }}$ e baixos valores de $\mathrm{S}_{\mathrm{yx}} \%$.
TABELA 1: Matriz de correlação linear simples entre as variáveis independentes correlacionadas com as variáveis dependentes carbono orgânico total da parte aérea $(\mathrm{CT})$, logaritmo neperiano do carbono orgânico total da parte aérea $(\operatorname{lnCT})$, carbono orgânico da lenha (CL) e logaritmo neperiano do carbono orgânico da lenha (lnCL).

TABLE 1: Linear correlation matrix between independent variables correlated with the dependent variables total above ground organic carbon (CT), natural logarithmic of total above ground organic carbon $(\operatorname{lnCT})$, firewood organic carbon (CL) and natural logarithmic of firewood organic carbon (lnCL).

\begin{tabular}{|c|c|c|c|c|}
\hline $\begin{array}{l}\text { Variável } \\
\text { independente }\end{array}$ & CT & $\operatorname{lnCT}$ & CL & $\operatorname{lnCL}$ \\
\hline $\mathrm{G}^{2} \overline{\mathrm{d}}$ & 0,9622 & 0,8818 & 0,9462 & 0,8575 \\
\hline $\mathrm{G}^{2} \mathrm{~d}_{\mathrm{g}}$ & 0,9610 & 0,8798 & 0,9440 & 0,8544 \\
\hline $\ln G^{2} \bar{d}$ & 0,9515 & 0,9842 & 0,9228 & 0,9385 \\
\hline $\ln G^{2} d_{g}$ & 0,9510 & 0,9833 & 0,9215 & 0,9365 \\
\hline $\mathrm{G} \overline{\mathrm{h}}$ & 0,9411 & 0,9135 & 0,9231 & 0,8842 \\
\hline $\mathrm{G}^{2} \overline{\mathrm{h}}$ & 0,9402 & 0,8673 & 0,8908 & 0,8097 \\
\hline $\mathrm{G}^{2} \mathrm{~h}_{\mathrm{dom}}$ & 0,9203 & 0,8481 & 0,8678 & 0,7868 \\
\hline $\operatorname{lnG\overline {h}}$ & 0,9131 & 0,9488 & 0,8873 & 0,9065 \\
\hline $\ln G^{2} \bar{h}$ & 0,9128 & 0,9549 & 0,8552 & 0,8739 \\
\hline $\mathrm{Gh}_{\mathrm{dom}}$ & 0,9122 & 0,8852 & 0,8880 & 0,8480 \\
\hline $\ln G^{2} h_{d o m}$ & 0,9001 & 0,9435 & 0,8398 & 0,8583 \\
\hline $\mathrm{Gd}_{\mathrm{g}}$ & 0,8894 & 0,8514 & 0,9270 & 0,8824 \\
\hline $\operatorname{lnGh} h_{\text {dom }}$ & 0,8879 & 0,9261 & 0,8561 & 0,8749 \\
\hline $\mathrm{G} \overline{\mathrm{d}}$ & 0,8873 & 0,8504 & 0,9263 & 0,8832 \\
\hline $\ln G$ & 0,8554 & 0,9018 & 0,7679 & 0,7848 \\
\hline G & 0,8924 & 0,8721 & 0,8010 & 0,7629 \\
\hline $\mathrm{h}_{\mathrm{dom}}$ & 0,2183 & 0,2083 & 0,3193 & 0,3232 \\
\hline$\overline{\mathrm{h}}$ & 0,1942 & 0,1757 & 0,3110 & 0,3133 \\
\hline $\mathrm{N}$ & 0,1645 & 0,1810 & $-0,0084$ & $-0,0264$ \\
\hline$d_{g}$ & 0,0394 & $-0,0034$ & 0,1790 & 0,1639 \\
\hline$\overline{\mathrm{d}}$ & 0,0315 & $-0,0114$ & 0,1718 & 0,1573 \\
\hline
\end{tabular}

Em que: $\mathrm{G}=$ Área basal por hectare $\left(\mathrm{m}^{2} \cdot \mathrm{ha}^{-1}\right) ; \overline{\mathrm{h}}=$ Altura total média $(\mathrm{m}) ; \mathrm{h}_{\mathrm{dom}}=$ Altura dominante $(\mathrm{m}) ; \overline{\mathrm{d}}$ $=$ Diâmetro a altura do peito médio $(\mathrm{cm}) ; \mathrm{d}_{\mathrm{g}}=$ Diâmetro médio quadrático $(\mathrm{cm}) ; \mathrm{N}=$ Número de indivíduos $(\mathrm{N} / \mathrm{ha}) ; \ln =$ Logaritmo neperiano. 
TABELA 2: Equações tradicionais ajustadas com respectivas estatísticas de ajuste e precisão para estimativa do carbono orgânico total da parte aérea $\left(\mathrm{kg}_{\mathrm{g}} \cdot \mathrm{ha}^{-1}\right)$.

TABLE 2: Traditional adjusted equations with respective statistics for total above ground organic carbon estimative $\left(\mathrm{kg} \cdot \mathrm{ha}^{-1}\right)$.

\begin{tabular}{|c|c|c|c|c|c|}
\hline Equação & Ajuste & $\mathrm{R}_{\mathrm{aj}}^{2}$ & $\mathrm{~S}_{\mathrm{yx}} \%$ & $\mathrm{~F}$ & FCM \\
\hline a1 & $\mathrm{CT}=1722,8626+1926,3234 \mathrm{G}$ & 0,7956 & 16,05 & 989 & - \\
\hline a2 & $\mathrm{CT}=2499,4897+148,9245 \mathrm{G} \overline{\mathrm{h}}$ & 0,8852 & 12,03 & 1960 & - \\
\hline a3 & $\mathrm{CT}=3892,6466+125,7867 \mathrm{Gh}_{\mathrm{dom}}$ & 0,8314 & 14,57 & 1254 & - \\
\hline a4 & $\mathrm{CT}=11727,4268+6,6299 \mathrm{G}^{2} \overline{\mathrm{h}}$ & 0,8835 & 12,11 & 1928 & - \\
\hline a5 & $\mathrm{CT}=12244,8849+5,7213 \mathrm{G}^{2} \mathrm{~h}_{\mathrm{dom}}$ & 0,8464 & 13,91 & 1401 & - \\
\hline$a 6^{\mathrm{A}}$ & $\mathrm{CT}=6,1362+\mathrm{G}^{0,9440}+\overline{\mathrm{h}}^{0,6493}$ & 0,9162 & 10,28 & 1341 & 1,0062 \\
\hline a7 & $\mathrm{CT}=6,4271+\mathrm{G}^{0,9204}+\mathrm{h}_{\mathrm{dom}}^{0,5354}$ & 0,8817 & 12,21 & 916 & 1,0085 \\
\hline a8 & $\mathrm{CT}=-67734,7695+18746,2076 \operatorname{lnG} \overline{\mathrm{h}}$ & 0,8332 & 14,50 & 1270 & - \\
\hline a9 & $\mathrm{CT}=-64012,3162+17637,2327 \mathrm{lnGh} \mathrm{dom}_{\mathrm{d}}$ & 0,7876 & 16,36 & 943 & - \\
\hline a10 & $\mathrm{CT}=-48227,8262+9930,7293 \operatorname{lnG}^{2} \overline{\mathrm{h}}$ & 0,8325 & 14,53 & 1264 & - \\
\hline $\ln 1$ & $\operatorname{lnCT}=7,8766+0,8989 \operatorname{lng}$ & 0,7946 & 16,09 & 940 & 1,0146 \\
\hline $\ln 2$ & $\operatorname{lnCT}=5,6366+0,8948 \ln \mathrm{Gh}_{\overline{\mathrm{h}}}$ & 0,8858 & 12,00 & 1999 & 1,0078 \\
\hline $\ln 3$ & $\operatorname{lnCT}=5,7983+0,8450 \operatorname{lnGh} h_{\text {dom }}$ & 0,8329 & 14,51 & 1317 & 1,0111 \\
\hline $\ln 4$ & $\operatorname{lnCT}=6,5440+0,4772 \ln G^{2} \overline{\mathrm{h}}$ & 0,9111 & 10,59 & 2462 & 1,0068 \\
\hline $\ln 5$ & $\operatorname{lnCT}=6,5973+0,4638 \ln \mathrm{G}^{2} \mathrm{~h}_{\mathrm{dom}}$ & 0,8824 & 12,17 & 1825 & 1,0085 \\
\hline $\ln 6$ & $\operatorname{lnCT}=5,6399+1,0091 \operatorname{lnG} \overline{\bar{h}}-0,1128 \operatorname{lnGh} \mathrm{dom}_{\mathrm{d}}$ & 0,8864 & 11,96 & 1006 & 1,0077 \\
\hline $\ln 7$ & $\operatorname{lnCT}=8,1787+0,1944 \ln G N$ & 0,2409 & 30,93 & 116 & 1,0532 \\
\hline $\ln 8$ & $\operatorname{lnCT}=7,9419+0,1753 \ln \mathrm{G}^{2} \mathrm{~N}$ & 0,3723 & 28,12 & 184 & 1,0429 \\
\hline $\ln 9$ & $\operatorname{lnCT}=9,6817+0,0470 \ln \overline{\mathrm{d}}^{2} \overline{\mathrm{h}}$ & 0,0107 & 35,31 & 3 & 1,0798 \\
\hline $\ln 10$ & $\operatorname{lnCT}=6,3535+0,3890 \ln N \bar{d}$ & 0,2399 & 30,95 & 114 & 1,0533 \\
\hline $\ln 11^{\mathrm{A}}$ & $\operatorname{lnCT}=7,7612+0,0161 \mathrm{~S}-2,9246 \mathrm{I}^{-1}+1,0134 \ln \mathrm{G}$ & 0,9166 & 10,25 & 912 & 1,0056 \\
\hline
\end{tabular}

Em que: $\mathrm{R}^{2}{ }_{\mathrm{aj}}=$ Coeficiente de determinação ajustado; $\mathrm{S}_{\mathrm{yx}} \% \frac{1}{-1}=$ Erro padrão da estimativa percentual; $\mathrm{F}=$ Teste $\mathrm{F} ; \mathrm{FCM}$ $=$ Fator de Correção de Meyer; $\mathrm{G}=$ Área basal $\left(\mathrm{m}^{2} \cdot \mathrm{ha}^{-1}\right) ; \overline{\mathrm{h}}=$ Altura total média $(\mathrm{m}) ; \mathrm{h}_{\text {dom }}=$ Altura dominante $(\mathrm{m}) ; \overline{\mathrm{d}}=$ Diâmetro médio a altura do peito (cm); $\mathrm{N}=$ Número de indivíduos $\left(\mathrm{N} \cdot \mathrm{ha}^{-1}\right) ; \mathrm{S}=$ Índice de sítio; I = idade (anos); ln = logaritmo neperiano; ${ }^{\mathrm{A}}=$ Modelos selecionados de melhor ajuste para cada caso.

No desenvolvimento do modelo aritmético pelo processo Stepwise, a variável $\mathrm{G}^{2} \overline{\mathrm{d}}$ foi a primeira a ingressar, e na sequência $\ln \mathrm{G}^{2} \overline{\mathrm{d}}, \mathrm{N}^{2}$ e $\mathrm{G}^{2}$. A equação final ajustada (3) apresentou $\mathrm{R}_{\text {aj }}$ igual a $0,9949, \mathrm{~S}_{\mathrm{yx}} \%$ igual a 2,53 e valor de $\mathrm{F}$ igual a 12458 .

$$
\begin{gathered}
\mathrm{CT}=-19894,2432+0,9697 \mathrm{G}^{2} \overline{\mathrm{d}}+ \\
5207,5765 \ln ^{2} \overline{\mathrm{d}}-0,0004 \mathrm{~N}^{2}+47,8022 \mathrm{G}^{2}
\end{gathered}
$$

Onde,

$\mathrm{CT}=$ Carbono orgânico total da parte aérea $\left(\mathrm{kg} \cdot \mathrm{ha}^{-1}\right) ; \mathrm{G}=$ Área basal $\left(\mathrm{m}^{2} \cdot \mathrm{ha}^{-1}\right) ; \overline{\mathrm{d}}=$ Diâmetro médio a altura do peito $(\mathrm{cm}) ; \mathrm{N}=$ Número de indivíduos (N.ha-1).

No caso do modelo logarítmico desenvolvido pelo processo Stepwise, o valor de $\mathrm{R}^{2}{ }_{\text {aj }}$ para a equação final ajustada (4) foi igual a 0,9958, com $\mathrm{S}_{\mathrm{yx}} \%$ de 2,30 e valor de $\mathrm{F}$ igual a 15279 . O Fator de Correção de Meyer calculado para esta equação foi 1,0005 .

$$
\begin{gathered}
\operatorname{lnCT}=6,2156+0,54891 \operatorname{lnG}^{2} \overline{\mathrm{d}}- \\
0,0000000173 \mathrm{~N}^{2}-0,000068 \mathrm{Gd}^{2}+ \\
0,00000035 \mathrm{GNd}_{\mathrm{g}}
\end{gathered}
$$

Em que: $\operatorname{lnCT}=$ Logaritmo neperiano do carbono orgânico total da parte aérea $\left(\mathrm{kg} \cdot \mathrm{ha}^{-1}\right) ; \mathrm{G}=$ Área basal $\left(\mathrm{m}^{2} \cdot \mathrm{ha}^{-1}\right) ; \overline{\mathrm{d}}=$ Diâmetro médio a altura do peito $(\mathrm{cm}) ; \mathrm{N}=$ Número de indivíduos $\left(\mathrm{N} \cdot \mathrm{ha}^{-1}\right)$; $\mathrm{d}_{\mathrm{g}}=$ Diâmetro quadrático $(\mathrm{cm})$.

$\mathrm{Na}$ Figura 1 podem ser observadas as distribuições de resíduos para as equações a6 e $\ln 11$, selecionadas dentre as tradicionais como mais acuradas, e para as equações semilogarítmica 
e logarítmicas desenvolvidas por Stepwise, respectivamente. A distribuição de resíduos para as 4 equações foi homogênea, independente e pouco dispersa ao longo da linha estimativa, o que dificultou a comparação gráfica. As demais equações tradicionais ajustadas apresentaram superestimativas para valores inferiores a $20.000 \mathrm{~kg} \cdot \mathrm{ha}^{-1}$ de carbono orgânico total da parte aérea.

A equação $\ln 11$, referente ao modelo proposto por Clutter, foi selecionada como a mais acurada dentre as tradicionais pela superioridade que apresentou nas estatísticas de ajuste e precisão, sendo graficamente muito semelhante à equação a6. Dentre as equações ajustadas por Stepwise, graficamente não se observou diferença expressiva, sendo a equação logarítmica considerada a mais acurada, devido ao melhor resultado encontrado para $\mathrm{R}_{\text {aj }}^{2}, \mathrm{~S}_{\mathrm{yx}}$ \% e valor de $\mathrm{F}$.

Ambas as equações selecionadas foram validadas pelo teste de aderência de Qui-quadrado, com valores de $x^{2}$ igual a 0,5467 e 0,0514, respectivamente para a equação $\ln 11$ e Stepwise, inferiores ao valor tabelado de 26,30. Graficamente foi possível confirmar a validade destas equações, sendo a dispersão de resíduos para as 17 unidades amostrais homogênea, independente e pouco dispersa ao longo da linha estimativa.

A seleção da equação $\ln 11$ foi um importante resultado, uma vez que este modelo tem como variáveis independentes a idade, o sítio e a área basal, podendo assim, ser utilizado um método alternativo ao método de parcelas com área fixa. O método de Bitterlich, por exemplo, caracterizase pela rapidez, praticidade e baixo custo no que se refere ao trabalho de campo, devendo ser considerado como uma alternativa nos inventários pré-corte dos bracatingais.

O modelo de Clutter é muito aplicado no meio florestal; Dias et al. (2005) aplicaram este modelo para predizer o crescimento em volume de povoamentos desbastados de eucalipto, encontrando resultados satisfatórios no ajuste do modelo. Em seus resultados esses autores apresentaram os resultados do ajuste com $\mathrm{R}^{2}$ igual a 0,73 e $\mathrm{S}_{\mathrm{yx}} \%$ de $19,38 \%$. Couto et al. (1989) também aplicaram o modelo de Clutter para a prognose do volume de povoamentos de Eucalipto uma vez que, este modelo, apresentou os melhores resultados quando comparado ao modelo proposto por Schumacher.
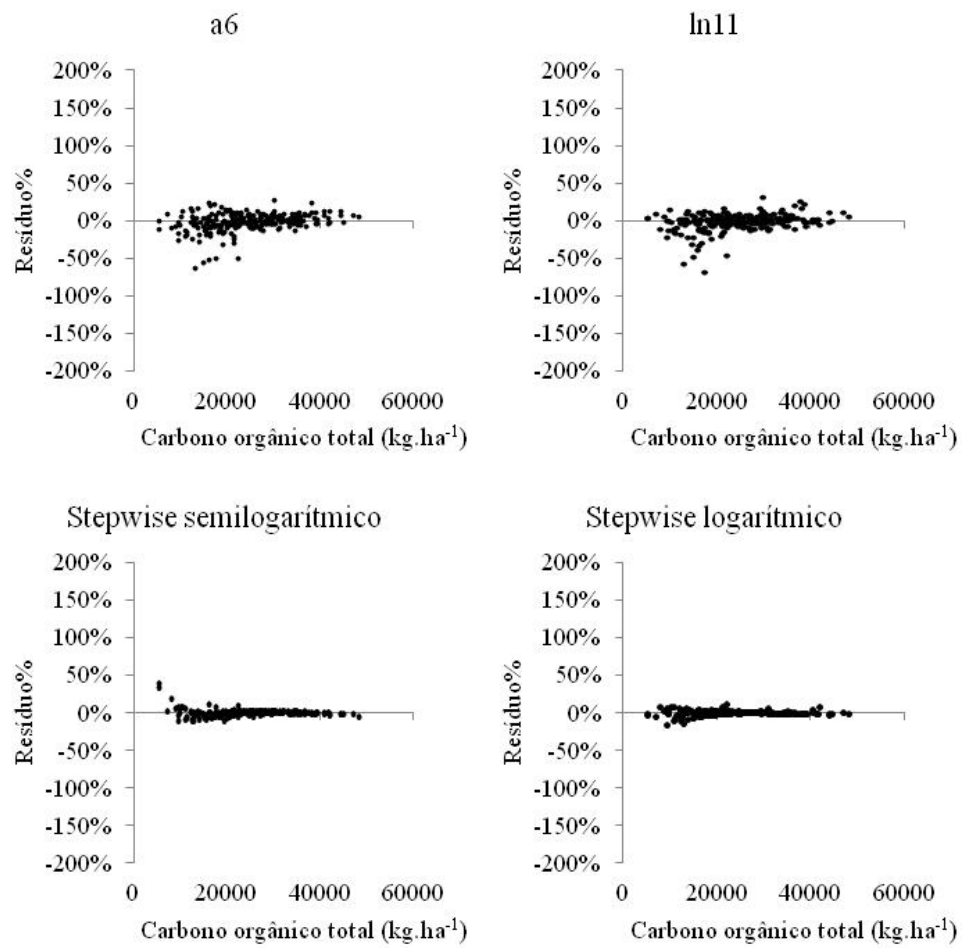

FIGURA 1: Dispersão de resíduos percentual do carbono orgânico total da parte aérea $\left(\mathrm{kg} \cdot \mathrm{ha}^{-1}\right)$ para as equações a6, $\ln 11$ e as equações semilogarítmica e logarítmica, desenvolvidas por Stepwise.

FIGURE 1: Residual dispersion for total above ground organic carbon $\left(\mathrm{kg} \cdot \mathrm{ha}^{-1}\right)$ for equations a6, $\ln 11$ and arithmetic and logarithmic equations developed by Stepwise. 
Trabalhos sobre carbono orgânico, em geral, utilizam metodologia onde os valores por unidade de área resultam da extrapolação de valores individuais que, por sua vez, são estimados por meio de equações ajustadas (BALBINOT et al., 2003; SOARES et al., 2005; URBANO et al., 2008). Assim, não houve a possibilidade de comparar os resultados obtidos com os ajustes dos modelos tradicionais.

\section{Equações estimativas do carbono orgânico da lenha}

Os resultados obtidos para o ajuste dos modelos tradicionais para estimativa do carbono orgânico da lenha foram satisfatórios. Elevados valores de $\mathrm{R}_{\text {aj }}$ e baixos valores de $\mathrm{S}_{\mathrm{yx}} \%$ foram observados tanto nas equações aritméticas e semilogarítmicas, quanto nas equações logarítmicas, excetuando-se as equações $\ln 7, \ln 8$, $\ln 9$ e $\ln 10$, como pode ser observado na Tabela 3.

No desenvolvimento do modelo aritmético pelo processo Stepwise, a variável independente que primeiro ingressou foi $\mathrm{G}^{2} \overline{\mathrm{d}}$, seguida por $\mathrm{N}^{2}$, $\mathrm{G}^{2} \mathrm{~d}_{\mathrm{g}}$ e $\mathrm{G}^{2}$. A equação final ajustada (5) apresentou $\mathrm{R}^{2}{ }_{\text {aj }}$ igual a $0,9918, \mathrm{~S}_{\mathrm{yx}} \%$ de 3,47 e valor de $\mathrm{F}$ igual a 7672 .

$$
\begin{aligned}
\mathrm{CL}= & -17869,4579+1,8191 \mathrm{G}^{2} \overline{\mathrm{d}}-0,0005 \mathrm{~N}^{2}+ \\
& 4532,9445 \ln \mathrm{G}^{2} \mathrm{~d}_{\mathrm{g}}+32,2725 \mathrm{G}^{2} \quad(5)
\end{aligned}
$$

Em que: $\mathrm{CL}=$ Carbono orgânico da lenha $\left(\mathrm{kg} \cdot \mathrm{ha}^{-1}\right) ; \mathrm{G}=$ Área basal $\left(\mathrm{m}^{2} \cdot \mathrm{ha}^{-1}\right) ; \overline{\mathrm{d}}=$ Diâmetro total médio $(\mathrm{cm}) ; \mathrm{N}=$ Número de indivíduos $\left(\mathrm{N} \cdot \mathrm{ha}^{-1}\right) ; \mathrm{d}_{\mathrm{g}}=$ Diâmetro Quadrático $(\mathrm{cm})$.

TABELA 3: Equações tradicionais ajustadas com respectivas estatísticas de ajuste e precisão para estimativa do carbono orgânico da lenha $\left(\mathrm{kg} \cdot \mathrm{ha}^{-1}\right)$.

TABLE 3: Traditional equations adjusted with respective statistics of fitting and precision for firewood

\begin{tabular}{|c|c|c|c|c|c|}
\hline Equação & Ajuste & $\mathrm{R}_{\mathrm{aj}}^{2}$ & $\mathrm{~S}_{\mathrm{yx}} \%$ & $\mathrm{~F}$ & FCM \\
\hline a1 & $\mathrm{CL}=2049,3379+1550,2443 \mathrm{G}$ & 0,6402 & 22,94 & 453 & - \\
\hline $\mathrm{a} 2^{\mathrm{A}}$ & $\mathrm{CL}=1061,3404+130,9740 \mathrm{G} \overline{\mathrm{h}}$ & 0,8516 & 14,73 & 1459 & - \\
\hline a3 & $\mathrm{CL}=2420,4303+109,7916 \mathrm{Gh}_{\mathrm{dom}}$ & 0,7878 & 17,62 & 944 & - \\
\hline $\mathrm{a} 4$ & $\mathrm{CL}=9548,3188+5,6317 \mathrm{G}^{2} \overline{\mathrm{h}}$ & 0,7926 & 17,42 & 972 & - \\
\hline a5 & $\mathrm{CL}=10035,6791+4,8368 \mathrm{G}^{2} \mathrm{~h}_{\mathrm{dom}}$ & 0,7521 & 19,04 & 771 & - \\
\hline a6 & $\mathrm{CL}=5,1573+\mathrm{G}^{0,9199}+\overline{\mathrm{h}}^{, 9835}$ & 0,8472 & 14,95 & 700 & 1,0165 \\
\hline a7 & $\mathrm{CL}=5,6079+\mathrm{G}^{0,8840}+\mathrm{h}_{\mathrm{dom}} 0,8072$ & 0,7920 & 17,44 & 477 & 1,0217 \\
\hline a8 & $\mathrm{CL}=-59950,4842+16332,1502 \ln \mathrm{G} \overline{\mathrm{h}}$ & 0,7865 & 17,67 & 937 & - \\
\hline a9 & $\mathrm{CL}=-56106,6348+15245,7231 \operatorname{lnG\mathrm {ndom}_{\mathrm {dom}}}$ & 0,7318 & 19,81 & 694 & - \\
\hline a10 & $\mathrm{CL}=-40699,6332+8342,1208 \ln G^{2} \overline{\mathrm{h}}$ & 0,7303 & 19,86 & 689 & - \\
\hline $\ln 1$ & $\operatorname{lnCL}=7,7934+0,8515 \ln G$ & 0,6384 & 23,00 & 419 & 1,0359 \\
\hline $\ln 2$ & $\operatorname{lnCL}=5,2651+0,9306 \operatorname{lnG\overline {h}}$ & 0,8510 & 14,76 & 1423 & 1,0165 \\
\hline $\ln 3$ & $\operatorname{lnCL}=5,4824+0,8690 \operatorname{lnGh} h_{\text {dom }}$ & 0,7876 & 17,63 & 943 & 1,0218 \\
\hline $\ln 4$ & $\operatorname{lnCL}=6,3613+0,4754 \ln G^{2} \overline{\mathrm{h}}$ & 0,8081 & 16,75 & 980 & 1,0219 \\
\hline $\ln 5$ & $\operatorname{lnCL}=6,4348+0,4592 \ln G^{2} h_{\text {dom }}$ & 0,7754 & 18,12 & 812 & 1,0245 \\
\hline $\ln 6^{\mathrm{A}}$ & $\operatorname{lnCL}=5,2760+1,3145 \operatorname{lnG} \overline{\hat{h}}-0,3787 \operatorname{lnGh} h_{\text {dom }}$ & 0,8540 & 14,61 & 733 & 1,0161 \\
\hline $\ln 7$ & $\operatorname{lnCL}=8,4389+0,1462 \operatorname{lnGN}$ & 0,1214 & 35,85 & 58 & 1,0804 \\
\hline $\ln 8$ & $\operatorname{lnCL}=8,1202+0,1437 \operatorname{lnG}^{2} \mathrm{~N}$ & 0,2232 & 33,71 & 90 & 1,0704 \\
\hline $\ln 9$ & $\operatorname{lnCL}=8,7557+0,1485 \ln ^{2} \overline{\mathrm{h}}$ & 0,0572 & 37,13 & 27 & 1,0885 \\
\hline $\ln 10$ & $\operatorname{lnCL}=7,0634+0,2929 \operatorname{lnN\overline {d}}$ & 0,1208 & 35,86 & 51 & 1,0804 \\
\hline $\ln 11$ & $\operatorname{lnCL}=7,6291+0,0233 \mathrm{~S}-4,4480 \mathrm{I}^{-1}+1,0270 \ln \mathrm{G}$ & 0,8524 & 14,69 & 495 & 1,0148 \\
\hline
\end{tabular}
organic carbon estimate $\left(\mathrm{kg} \cdot \mathrm{ha}^{-1}\right)$.

Em que: $\mathrm{R}^{2} \mathrm{aj}=$ Coeficiente de determinação ajustado; $\mathrm{Syx} \%=$ Erro padrão da estimativa percentual; $\mathrm{F}=$ Teste F; FCM

$=$ Fator de Correção de Meyer; $\mathrm{G}=$ Área basal $\left(\mathrm{m}^{2} \cdot \mathrm{ha}^{-1}\right) ; \overline{\mathrm{h}}=$ Altura total média $(\mathrm{m}) ; \mathrm{h}_{\text {dom }}=$ Altura dominante $(\mathrm{m}) ; \overline{\mathrm{d}}=$ Diâmetro médio a altura do peito $(\mathrm{cm}) ; \mathrm{N}=$ Número de indivíduos $\left(\mathrm{N} \cdot \mathrm{ha}^{-1}\right) ; \mathrm{S}=$ Índice de sítio; $\mathrm{I}=$ idade (anos); $\ln =$ logaritmo neperiano; ${ }^{\mathrm{A}}=$ Modelos selecionados de melhor ajuste para cada caso. 
A equação logarítmica final ajustada (6), desenvolvida pelo processo Stepwise para a estimativa do carbono orgânico da lenha, apresentou valor de $\mathrm{R}_{\text {aj }}^{2}$ igual a $0,9798, \mathrm{~S}_{\mathrm{yx}} \%$ de 5,43 e valor de F de 3530. O Fator de Correção de Meyer calculado foi de 1,0016 .

$$
\begin{aligned}
\operatorname{lnCL}= & 6,4069+0,4880 \ln \mathrm{G}^{2} \overline{\mathrm{d}}-0,00000004 \mathrm{~N}^{2}+ \\
& 0,00001298 \mathrm{GN}-0,0001 \mathrm{~N}
\end{aligned}
$$

Em que: $\operatorname{lnCL}=$ Logaritmo neperiano do carbono orgânico da lenha (kg.ha- $\left.{ }^{-1}\right) ; \mathrm{G}=$ Área basal $\left(\mathrm{m}^{2} \cdot \mathrm{ha}^{-1}\right) ; \overline{\mathrm{d}}=$ Diâmetro médio a altura do peito $(\mathrm{cm})$; $\mathrm{N}=$ Número de indivíduos $\left(\mathrm{N} \cdot \mathrm{ha}^{-1}\right)$.

$\mathrm{Na}$ Figura 2 pode ser observada a distribuição de resíduos percentuais do carbono orgânico da lenha para as equações a2 e ln6, selecionadas dentre as equações tradicionais aritméticas e logarítmicas, e para as equações desenvolvidas por Stepwise para ambos os casos.

Uma leve tendência de superestimativas para valores inferiores a $20.000 \mathrm{~kg} \cdot \mathrm{ha}^{-1}$ foi observada para as equações tradicionais, dentre as quais, a equação $\ln 6$, referente ao modelo proposto por Ung e Ouellet, foi selecionada como mais acurada devido
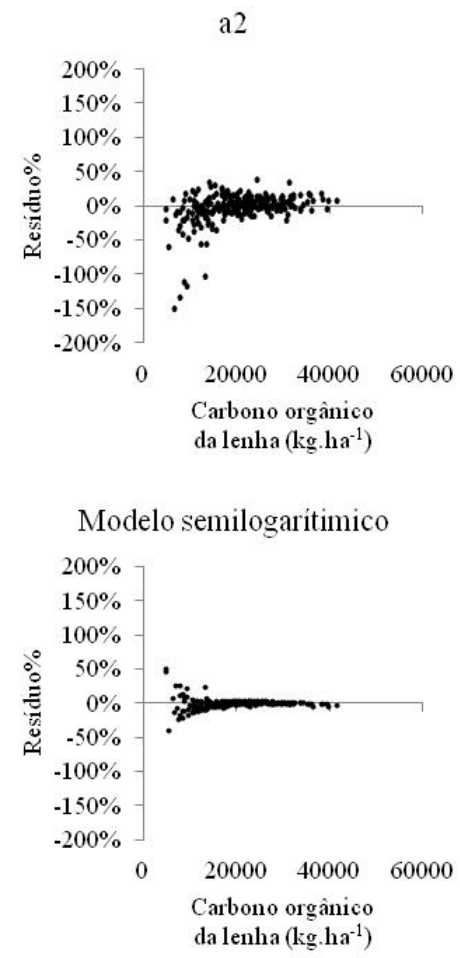

à melhor estatística de ajuste que apresentou. Dentre as equações desenvolvidas por Stepwise, também foi observada levesuperestimativa para valores inferiores a $20.000 \mathrm{~kg} \cdot \mathrm{ha}^{-1}$ para a equação logarítmica, sendo selecionada a equação semilogarítmica como de melhor ajuste. Destaca-se que a estatística de ajuste dessa equação foi superior à da equação logarítmica.

Ambas as equações foram consideradas válidas pelo teste de Qui-quadrado, com valores de $x^{2}$ iguais a 2,1367 e 0,1333 , respectivamente para a equação $\ln 6$ e Stepwise semilogarítmica. O valor tabelado de $x^{2}$ foi igual a 26,30. A análise gráfica das 17 parcelas separadas da base de dados comprovou também a validade destas equações, na qual, a distribuição de resíduos foi homogênea, independente e pouco dispersa ao longo da linha estimativa.

A equação $\ln 11$, referente ao modelo proposto por Clutter, ajustada para a estimativa do carbono orgânico da lenha apresentou $\mathrm{R}_{\text {aj }}^{2}$ igual a 0,8524 e $\mathrm{S}_{\mathrm{yx}} \%$ de 14,69 . Este resultado merece destaque, pois, como mencionado anteriormente, esta equação permite que a amostragem seja realizada pelo método de Bitterlich em inventários pré-corte.
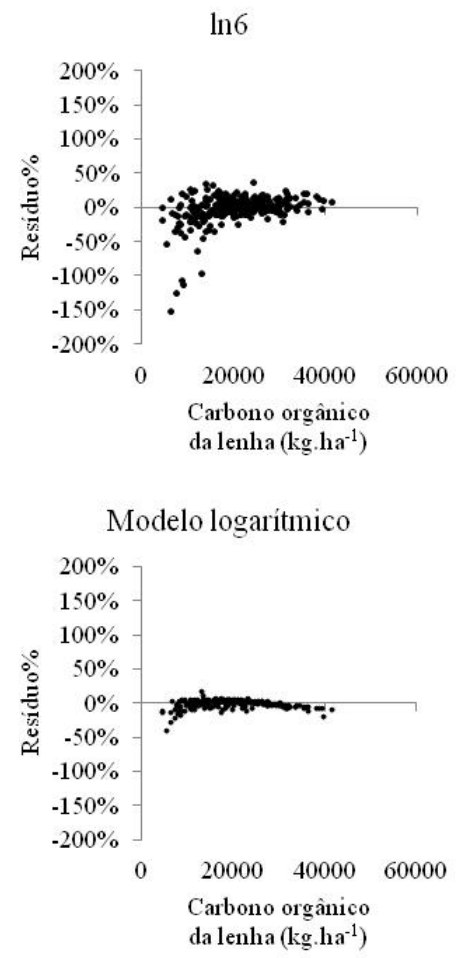

FIGURA 2: Dispersão de resíduos percentual do carbono orgânico da lenha (kg.ha-1 ${ }^{-1}$ para as equações a2, $\ln 6$ e as equações aritmética e logarítmica, construídas pelo processo Stepwise.

FIGURE 2: Residual dispersion of firewood organic carbon $\left(\mathrm{kg} \cdot \mathrm{ha}^{-1}\right)$ for equations a2, $\ln 6$ and the arithmetic and the logarithmic equations developed by Stepwise. 
O número de indivíduos, na sua forma pura ou transformada, foi uma variável que apresentou baixa correlação linear simples com as variáveis dependentes, no entanto, foi selecionada como variável independente em todos os modelos construídos por Stepwise. Justificou-se este fato pelo alto coeficiente de variação que esta variável apresentou, quando analisada a base de dados, bem como, a baixa correlação que esta variável tem com as demais variáveis do povoamento, sendo assim, significativa para a melhoria do ajuste das equações finais.

A seleção de uma equação aritmética como mais acurada, como foi o caso da equação ajustada por Stepwise para o carbono orgânico da lenha, contrariou resultados encontrados por diversos autores como Figueiredo Filho et al. (1982); Rosot et al. (1990); Silva (1995); Oliveira et al. (2005) e Machado et al. (2008). Esses autores destacaram em seus resultados que, modelos logarítmicos são mais eficientes, pois, com a transformação das variáveis ocorre a diminuição da escala e, consequentemente menor variação, o que permite um melhor ajuste dos modelos.

A bracatinga, na sua forma tradicional de cultivo, apresenta uma variabilidade muito expressiva, o que dificulta a homogeneização dos dados, mesmo quando logaritmizados. Assim, modelos aritméticos e semilogarítmicos podem apresentar resultados melhores em determinadas situações que modelos logarítmicos, como foi o caso dos modelos desenvolvidos por Stepwise para estimativa do carbono orgânico da lenha.

As equações ajustadas para o volume por unidade de área apresentam entre as variáveis independentes, combinações que envolvem a altura total média e altura dominante; esse mesmo resultado foi encontrado por diversos autores como Figueiredo Filho et al. (1982); Rosot et al. (1990); Silva (1995); Oliveira et al. (2005) e Machado et al. (2008). Este resultado não foi observado para as equações ajustadas pelo método Stepwise, selecionadas para a estimativa do carbono orgânico total da parte aérea e da lenha dos bracatingais da região metropolitana de Curitiba. Este resultado já era esperado, pois, na matriz de correlação apresentada na Tabela 1 as variáveis mais correlacionadas com as variáveis dependentes não continham a altura. Urbano et al. (2008) também selecionaram equações estimativas do carbono em indivíduos de bracatinga que não continham a variável altura.

\section{CONCLUSÕES}

Acorrelação linear simples entre as variáveis utilizadas diferenciou-se daqueles resultados tradicionalmente encontrados para o volume dos povoamentos. Este resultado foi também constatado no ajuste dos modelos matemáticos por Stepwise, nos quais, a variável altura nas suas diferentes formas não compôs nenhum dos modelos ajustados.

Apesar de eficientes, as equações tradicionais selecionadas mostraram-se menos acuradas que as equações ajustadas por Stepwise, reforçando a importância e a melhoria que este método representa para estudos de modelagem.

As equações ajustadas, referentes ao modelo de Clutter, são viáveis de aplicação nas estimativas do carbono orgânico total da parte aérea e da lenha dos bracatingais, sendo necessária apenas sua validação antes do uso. Estas equações ajustadas são de grande valia em termos práticos para os produtores da região, uma vez que, permitem a aplicação do método de Bitterlich nos inventários pré-corte, tornando o trabalho rápido, prático e de baixo custo.

Sugere-se o desenvolvimento de trabalhos no que se refere à quantificação do carbono orgânico armazenado nas raízes e no solo dos povoamentos de bracatinga, servindo de complemento a este trabalho.

\section{REFERÊNCIAS BIBLIOGRÁFICAS}

ALMEIDA, E. M.; JUNIOR, J. H. C.; FINGER, Z. Determinação do estoque de carbono em Teca (Tectona grandis F. F.) em diferentes idades. Ciência Florestal, Santa Maria, v. 20, n. 4, p. 559568, out.-dez., 2010.

BALBINOT, R. et al. Inventário do carbono orgânico em um plantio de Pinus taeda aos 5 anos de idade no Rio Grande do Sul. Ciências Exatas e Naturais. v. 5, no 1, jan/jun 2003.

CARPANEZZI, A. A. et al. Manual Técnico da Bracatinga (Mimosa scabrella Bentham). Curitiba: EMBRAPA-CNPF, 1988. 70 p. (Série Documentos, n.20).

CARPANEZZI, A. A.; CARPANEZZI, O. T. B. Cultivo da bracatinga (Mimosa scabrella Bentham) no Brasil e prioridades para o seu aperfeiçoamento. In: CONGRESSO FLORESTAL ESTADUAL, 7., 1992, Nova Prata. Anais... Santa Maria: Ed. da UFSM, 1992.2 v., p.640-655.

COUTO, H. T. Z.; BASTOS, N. L. M.; LACERDA, 
N. L. S. Comparação de dois modelos de prognose de madeira de Eucalyptus para alto fuste e talhadia. IPEF, n.41/42, p.27-35, jan./dez.1989.

DIAS, A. N. et al. Emprego de um povoamento de crescimento e produção em povoamentos desbastados de eucalipto. Revista Árvore, Viçosa, v. 29 , n. 5, p. 731-739, 2005.

FIGUEIREDO FILHO, A.; MACHADO, S. A.; ROSOT, N. C. Estimativas volumétricas por Unidade de Área em Florestas Naturais. Silvicultura Em São Paulo, São Paulo, v. 16, p. 491-504, 1982. GORDENS, E. B. et al. Metodologia para monitoramento do estoque de carbono: revisão e estudo de caso. Biomassa e Energia, v. 2, n. 2, p. 169-175, 2005.

MAACK, R. Geografia física do estado do Paraná. 3. ed. Curitiba: Imprensa Oficial do Paraná, 2002. $440 \mathrm{p}$.

MACHADO S. A. et al. Modelagem do volume do povoamento para Mimosa scabrella Benth. na região metropolitana de Curitiba. Revista Árvore, Viçosa, v. 32, n. 3, p. 465-478, 2008.

MACHADO, S. A. et al. Classificação de sítios para bracatingais nativos da região metropolitana de Curitiba. Cerne, Lavras, v. 17, n. 4, out./dez. 2011. OLIVEIRA, M. L. R. et al. Equações de volume de povoamento para fragmentos florestais naturais do município de Viçosa, Minas Gerais. Revista Árvore, Viçosa, v. 29, n. 2, p. 213-225, 2005.

ROCHADELLI, R. A estrutura de fixação dos átomos de carbono em reflorestamentos: Estudo de caso: Mimosa scabrella Bentham, bracatinga. 2001.86 f. Tese (Doutorado em Ciências Florestais), Universidade Federal do Paraná, Curitiba, 2001. ROSOT, M. A. D.; MACHADO, S. A.; ROSOT, N. C. . Modelos matemáticos para estimar o volume por hectare em um povoamento de Pinus taeda L. em Piraí do Sul - PR. In: CONGRESSO FLORESTAL BRASILEIRO, 1990, Campos do Jordão. Anais. São Paulo, 1990. v. 3. p. 126-138.

SANQUETTA, C. R. Métodos de determinação de biomassa florestal. In: carbono. Curitiba, 2002. p. 119-140.

SCHNEIDER, P. R. et al. Determinação indireta do estoque de biomassa e carbono em povoamentos de Acácia-negra (Acacia mearnsii De Wild.). Ciência Florestal, Santa Maria, v. 15, n. 4, p. 391-402, 2005. SILVA, J. de A. . Modelo de equação para estimar o volume por hectare de Eucalyptus spp na região de Campo Grande, no estado do Mato Grosso do Sul. Floresta e Ambiente, Seropédica, v. 2, p. 5159, 1995.

SOARES, C. P. B.; LEITE, H. G.; GORDENS, E. B. Equações para estimar o estoque de carbono no fuste de árvores individuais e em plantios comerciais de eucalipto. Revista Árvore, Viçosa, v. 29, n. 5, p. 711-718, 2005.

URBANO E. et al. Equações para estimar o peso de carbono fixado em árvores de Mimosa scabrella Bentham (BRACATINGA) em povoamentos nativos. Cerne, Lavras, v. 14, n. 3, p. 194-203, 2008.

WATZLAWICK, L. F. et al. Teores de carbono em espécies da Floresta Ombrófila Mista. Fixação de carbono: Atualidades, Projetos e Pesquisa. In: SIMPÓSIO LATINO AMERICANO SOBRE FIXAÇÃO DE CARBONO, 2., 2004, Curitiba. Anais... Curitiba, 2004. p. 95 - 109.

WEBER, K. S. et al. Teores de carbono orgânico de seis espécies naturais do ecossistema da Floresta Ombrófila Mista. Ambiência, Guarapuava, v. 2, n. 2, p. 167-177, jul./dez. 2006. 